\title{
Analysis of Orthogonal and Biorthogonal Wavelet Filters for Image Compression
}

\author{
Sarita Kumari \\ Deptt. of Physics \\ Banasthali University \\ Banasthali, India-304022
}

\author{
Ritu Vijay \\ Deptt. of Electronics \\ Banasthali University \\ Banasthali, India-304022
}

\begin{abstract}
In the present work we analyze the performance of orthogonal and Biorthogonal wavelet filters for image compression on variety of test images. The test images are of different size and resolution. The compression performance is measured, objectively peak signal to noise ratio and subjectively visual quality of image and it is found that Biorthogonal wavelets outperform the orthogonal ones in both the criteria.
\end{abstract}

\section{General Terms}

Image compression algorithms

\section{Keywords}

Wavelet transform; compression ratio; peak signal to noise ratio

\section{INTRODUCTION}

The computer is becoming more and more powerful day by day. As a result, the use of digital images is increasing rapidly. Along with this increasing use of digital images comes the serious issue of storing and transferring the huge volume of data representing the images because the uncompressed multimedia (graphics, audio and video) data requires considerable storage capacity and transmission bandwidth. Internet teleconferencing, High Definition Television (HDTV), satellite communications and digital storage of movies are not feasible without a high degree of compression. As it is, such applications are far from realizing their full potential largely due to the limitations of common image compression techniques [1], it is essential to find a new compression scheme with effective compression performance.

Wavelet transform is one of the promising methods of image compression. It has received significant attention recently due to their suitability for a number of image processing tasks including image compression [2] [3]. The basic measure of the performance of a compression algorithm is the compression ratio and peak signal to noise ratio, which is defined by the ratio between original data size and compressed data size. In the present work, we analyze various wavelet families for image compression on variety of test images and then compare the performance of wavelets. According to this analysis, we show the selection of the optimal wavelet for image compression taking into account Peak Signal to Noise Ratio (PSNR) as objective and visual quality of image as subjective quality measures.

\section{WAVELET FAMILIES}

Wavelet families can be divided into two main categories, orthogonal and Biorthogonal wavelets, which have different properties of basis functions. Orthogonality decorrelates the transform coefficients there by minimizing redundancy. Symmetry provides linear phase and minimize border arti-facts.
Other Important properties of wavelet functions in image compression applications are compact support, symmetry, regularity and degree of smoothness [4] [5]. Figure 1 illustrates some of the commonly used wavelet functions in our experiments.

\section{QUALITY MEASURES}

The performances of image compression techniques are mainly analyzed on the basis of two measures: Compression Ratio (CR) and Peak Signal to noise ratio (PSNR). The compression ratio is defined as ratio of the size of original data set to the size of the compressed data set [6].

Comprassion_ratio $=\frac{A}{B} \times 100$

Where $\mathrm{A}=$ Number of Bytes in the original data set $\mathrm{B}=$ Number of Bytes in the Compressed data set

PSNR provides a measurement of the amount of distortion in a signal, with a higher value indicating less distortion. For n-bits per pixel image, PSNR is defined as:

$$
P S N R=20 \log _{10} \frac{2^{R}-1}{R M S E} d b
$$

Where, RMSE is the root mean square difference between two images. The Mean Square Error (MSE) is defined as follows:

$$
M S E=\frac{1}{M N} \sum_{m=0}^{M-1} \sum_{n=0}^{N-1}|y(m, n)-x(m, n)|^{2}
$$

where $x(m, n), y(m, n)$ are respectively the original and recovered pixel values at the $\mathrm{m}^{\text {th }}$ row and $\mathrm{n}^{\text {th }}$ column for $\mathrm{MxN}$ size image. The PSNR is given in decibel units (Db), which measures the ratio of the peak signal and the error signal (difference between two images). The PSNR value provides the quality objectively. While, visual quality of image is considered as subjective quality measures.

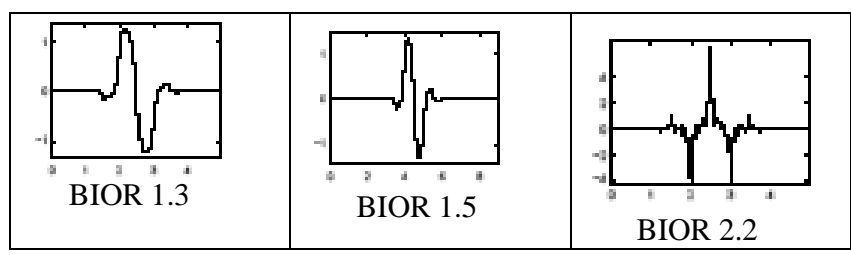




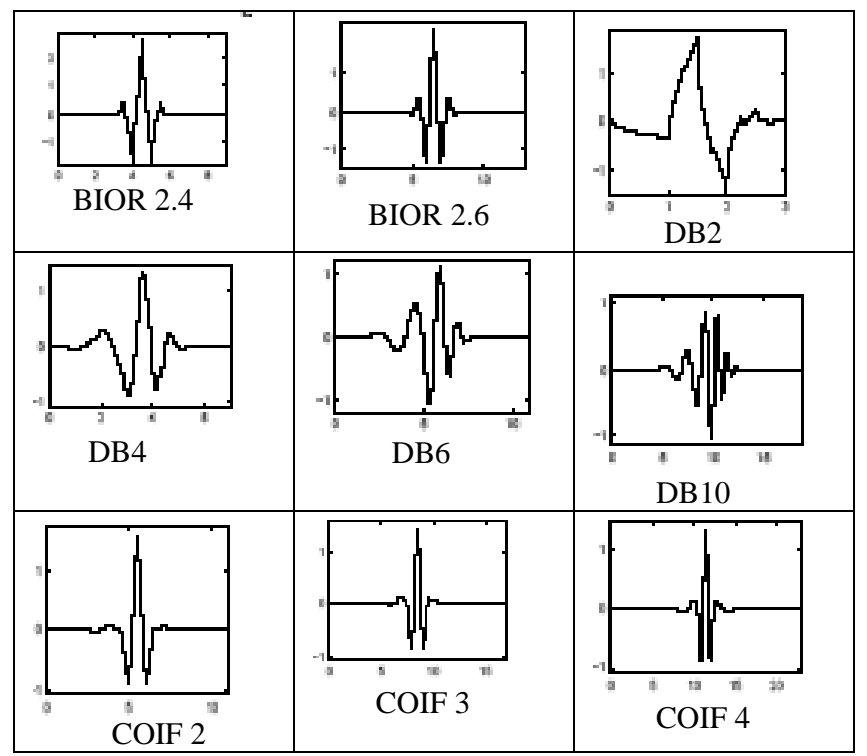

Figure 1: Wavelets families used in our experiments

\section{EXPERIMENTAL RESULTS, ANALYSIS AND COMPARISON}

We analyze orthogonal and Biorthogonal wavelet families for image compression and compare their results. We used four types of test images with different frequency content, different resolution and different size: Wbarb(512X512), Pepper(512X512), Bird(256X256) and Bridge(256X256). Results are shown in table 1 to table 4.

Table 1: Wavelet Family: Biorthogonal PSNR (in Db)

\begin{tabular}{|c|c|c|c|c|c|c|}
\hline \multicolumn{7}{|c|}{ PSNR (in Db) } \\
\hline Image & $\mathrm{CR}$ & $\begin{array}{l}\text { BIOR } \\
1.3\end{array}$ & $\begin{array}{l}\text { BIOR } \\
1.5\end{array}$ & $\begin{array}{l}\text { BIOR } \\
2.2\end{array}$ & $\begin{array}{l}\text { BIOR } \\
2.4\end{array}$ & $\begin{array}{l}\text { BIOR } \\
2.6\end{array}$ \\
\hline \multirow[t]{6}{*}{ Wbarb } & $10: 1$ & 42.07 & 41.94 & 45.01 & 45.21 & 45.27 \\
\hline & $20: 1$ & 36.50 & 36.35 & 39.50 & 39.70 & 39.71 \\
\hline & $40: 1$ & 31.24 & 31.15 & 34.29 & 34.54 & 34.46 \\
\hline & $60: 1$ & 28.42 & 28.32 & 31.39 & 31.68 & 31.60 \\
\hline & $80: 1$ & 26.52 & 26.42 & 29.47 & 29.74 & 29.67 \\
\hline & 100:1 & 25.11 & 25.00 & 28.05 & 28.29 & 28.23 \\
\hline \multirow[t]{6}{*}{ Pepper } & $10: 1$ & 42.07 & 41.98 & 44.79 & 45.18 & 45.29 \\
\hline & $20: 1$ & 36.54 & 36.43 & 39.34 & 39.63 & 39.72 \\
\hline & $40: 1$ & 31.35 & 31.20 & 34.15 & 34.43 & 34.47 \\
\hline & $60: 1$ & 28.49 & 28.34 & 31.27 & 31.56 & 31.59 \\
\hline & $80: 1$ & 26.52 & 26.40 & 29.32 & 29.61 & 29.60 \\
\hline & $100: 1$ & 25.06 & 24.97 & 27.90 & 28.19 & 28.11 \\
\hline \multirow[t]{6}{*}{ Bird } & $10: 1$ & 52.17 & 48.61 & 52.17 & 52.19 & 52.34 \\
\hline & $20: 1$ & 47.11 & 43.41 & 47.11 & 47.20 & 47.19 \\
\hline & $40: 1$ & 41.87 & 38.62 & 41.87 & 42.08 & 42.16 \\
\hline & $60: 1$ & 38.88 & 35.93 & 38.88 & 39.12 & 39.21 \\
\hline & $80: 1$ & 36.82 & 34.08 & 36.82 & 37.07 & 37.21 \\
\hline & $100: 1$ & 35.31 & 32.68 & 35.31 & 35.51 & 35.67 \\
\hline \multirow[t]{6}{*}{ Bridge } & $10: 1$ & 47.72 & 47.59 & 50.68 & 51.12 & 51.06 \\
\hline & $20: 1$ & 41.92 & 41.80 & 44.87 & 45.36 & 45.23 \\
\hline & $40: 1$ & 36.40 & 36.28 & 39.21 & 39.75 & 39.65 \\
\hline & $60: 1$ & 33.40 & 33.25 & 36.09 & 36.61 & 36.51 \\
\hline & $80: 1$ & 31.39 & 31.21 & 33.97 & 34.48 & 34.35 \\
\hline & 100:1 & 29.96 & 29.73 & 32.41 & 32.93 & 32.76 \\
\hline
\end{tabular}

Table 2: Wavelet Family: Daubechies

\begin{tabular}{|c|c|c|c|c|c|c|}
\hline \multicolumn{7}{|c|}{ PSNR (in Db) } \\
\hline Image & CR & DB 2 & DB 4 & DB 6 & $\begin{array}{l}\text { DB } \\
10\end{array}$ & DB 20 \\
\hline \multirow[t]{6}{*}{ Wbarb } & $10: 1$ & 42.15 & 42.03 & 41.92 & 41.87 & 41.61 \\
\hline & $20: 1$ & 36.71 & 36.65 & 36.45 & 36.35 & 35.95 \\
\hline & $40: 1$ & 31.65 & 31.67 & 31.41 & 31.25 & 30.74 \\
\hline & $60: 1$ & 28.93 & 29.00 & 28.70 & 28.56 & 28.01 \\
\hline & $80: 1$ & 27.14 & 27.25 & 26.91 & 26.81 & 26.26 \\
\hline & 100:1 & 25.84 & 25.97 & 25.63 & 25.56 & 25.01 \\
\hline \multirow[t]{6}{*}{ Pepper } & $10: 1$ & 42.05 & 41.99 & 41.90 & 41.74 & 41.55 \\
\hline & $20: 1$ & 36.63 & 36.55 & 36.38 & 36.13 & 35.85 \\
\hline & $40: 1$ & 31.53 & 31.54 & 31.19 & 30.85 & 30.43 \\
\hline & $60: 1$ & 28.75 & 28.75 & 28.35 & 27.99 & 27.46 \\
\hline & $80: 1$ & 26.86 & 26.88 & 26.46 & 26.07 & 25.52 \\
\hline & 100:1 & 25.46 & 25.52 & 25.06 & 24.67 & 24.12 \\
\hline \multirow[t]{6}{*}{ Bird } & $10: 1$ & 49.54 & 49.10 & 48.68 & 48.47 & 47.52 \\
\hline & $20: 1$ & 44.41 & 43.92 & 43.50 & 43.08 & 42.20 \\
\hline & $40: 1$ & 39.34 & 38.99 & 38.53 & 38.13 & 37.29 \\
\hline & $60: 1$ & 36.49 & 36.14 & 35.70 & 35.37 & 34.66 \\
\hline & $80: 1$ & 34.57 & 34.23 & 33.85 & 33.48 & 32.90 \\
\hline & 100:1 & 33.17 & 32.86 & 32.50 & 32.07 & 31.60 \\
\hline \multirow[t]{6}{*}{ Bridge } & $10: 1$ & 47.73 & 47.59 & 47.54 & 47.62 & 47.44 \\
\hline & $20: 1$ & 42.00 & 41.85 & 41.74 & 41.89 & 41.68 \\
\hline & $40: 1$ & 36.56 & 36.44 & 36.35 & 36.41 & 36.18 \\
\hline & $60: 1$ & 33.60 & 33.44 & 33.41 & 33.40 & 33.18 \\
\hline & $80: 1$ & 31.65 & 31.52 & 31.48 & 31.40 & 31.24 \\
\hline & $100: 1$ & 30.21 & 30.14 & 30.11 & 29.97 & 29.86 \\
\hline
\end{tabular}

Table 3: Wavelet Family: Coiflet

\begin{tabular}{|l|l|l|l|l|l|l|}
\hline \multicolumn{7}{|c|}{ PSNR (in Db) } \\
\hline Image & CR & COIF 1 & $\begin{array}{l}\text { COIF } \\
\mathbf{2}\end{array}$ & $\begin{array}{l}\text { COIF } \\
\mathbf{3}\end{array}$ & $\begin{array}{l}\text { COIF } \\
\mathbf{4}\end{array}$ & $\begin{array}{l}\text { COIF } \\
\mathbf{5}\end{array}$ \\
\hline \multirow{5}{*}{ Wbarb } & $10: 1$ & 42.16 & 42.06 & 42.14 & 42.04 & 42.13 \\
\cline { 2 - 7 } & $20: 1$ & 36.76 & 36.71 & 36.73 & 36.64 & 36.70 \\
\cline { 2 - 7 } & $40: 1$ & 31.71 & 31.75 & 31.79 & 31.62 & 31.66 \\
\cline { 2 - 7 } & $60: 1$ & 28.95 & 29.07 & 29.16 & 28.92 & 28.97 \\
\cline { 2 - 7 } & $80: 1$ & 27.16 & 27.28 & 27.40 & 27.15 & 27.19 \\
\cline { 2 - 7 } & $100: 1$ & 25.87 & 25.98 & 26.12 & 25.87 & 25.89 \\
\hline Pepper & $10: 1$ & 42.06 & 42.03 & 42.02 & 41.94 & 42.09 \\
\cline { 2 - 7 } & $20: 1$ & 36.65 & 36.61 & 36.59 & 36.46 & 36.62 \\
\cline { 2 - 7 } & $40: 1$ & 31.55 & 31.58 & 31.51 & 31.34 & 31.47 \\
\cline { 2 - 7 } & $60: 1$ & 28.76 & 28.89 & 28.73 & 28.54 & 28.66 \\
\cline { 2 - 7 } & $80: 1$ & 26.88 & 27.05 & 26.87 & 26.68 & 26.79 \\
\cline { 2 - 7 } & $100: 1$ & 25.48 & 25.69 & 25.49 & 25.33 & 25.43 \\
\hline Bird & $10: 1$ & 49.40 & 49.25 & 49.17 & 49.03 & 48.94 \\
\cline { 2 - 7 } & $20: 1$ & 44.34 & 44.07 & 44.04 & 44 & 43.81 \\
\cline { 2 - 7 } & $40: 1$ & 39.29 & 39.11 & 39.10 & 38.98 & 38.98 \\
\cline { 2 - 7 } & $60: 1$ & 36.47 & 36.46 & 36.39 & 36.16 & 36.19 \\
\cline { 2 - 7 } & $80: 1$ & 34.52 & 34.66 & 34.52 & 34.23 & 34.29 \\
\cline { 2 - 7 } & $100: 1$ & 33.06 & 33.28 & 33.14 & 32.78 & 32.93 \\
\hline \multirow{5}{*}{ Bridge } & $10: 1$ & 47.76 & 47.61 & 47.70 & 47.70 & 47.70 \\
\cline { 2 - 7 } & $20: 1$ & 42.03 & 41.88 & 41.97 & 41.94 & 41.94 \\
\cline { 2 - 7 } & $40: 1$ & 36.60 & 36.46 & 36.52 & 36.39 & 36.51 \\
\cline { 2 - 7 } & $60: 1$ & 33.63 & 33.56 & 33.53 & 33.43 & 33.54 \\
\cline { 2 - 6 } & $80: 1$ & 31.70 & 31.67 & 31.56 & 31.54 & 31.63 \\
\cline { 2 - 7 } & $100: 1$ & 30.2994 & 30.32 & 30.13 & 30.15 & 30.25 \\
\hline
\end{tabular}


Table 4: Wavelet Family: Symlet

\begin{tabular}{|c|c|c|c|c|c|c|}
\hline \multicolumn{7}{|c|}{ PSNR (in Db) } \\
\hline Image & CR & $\begin{array}{l}\text { SYM } \\
2\end{array}$ & $\begin{array}{l}\text { SYM } \\
\mathbf{3}\end{array}$ & $\begin{array}{l}\text { SYM } \\
5\end{array}$ & $\begin{array}{l}\text { SYM } \\
10\end{array}$ & $\begin{array}{l}\text { SYM } \\
12\end{array}$ \\
\hline \multirow{6}{*}{ Wbarb } & $10: 1$ & 41.97 & 42.12 & 42.10 & 42.08 & 42.03 \\
\hline & $20: 1$ & 36.48 & 36.77 & 36.73 & 36.63 & 36.57 \\
\hline & $40: 1$ & 31.34 & 31.74 & 31.77 & 31.67 & 31.56 \\
\hline & $60: 1$ & 28.55 & 29.02 & 29.12 & 29.01 & 28.89 \\
\hline & $80: 1$ & 26.70 & 27.22 & 27.35 & 27.26 & 27.12 \\
\hline & $100: 1$ & 25.36 & 25.92 & 26.08 & 25.97 & 25.85 \\
\hline \multirow[t]{6}{*}{ Pepper } & $10: 1$ & 42.08 & 42.03 & 42.01 & 41.96 & 41.95 \\
\hline & $20: 1$ & 36.65 & 36.59 & 36.54 & 36.46 & 36.40 \\
\hline & $40: 1$ & 31.48 & 31.52 & 31.46 & 31.33 & 31.18 \\
\hline & $60: 1$ & 28.60 & 28.73 & 28.69 & 28.53 & 28.38 \\
\hline & $80: 1$ & 26.64 & 26.87 & 26.84 & 26.67 & 26.53 \\
\hline & 100:1 & 25.21 & 25.51 & 25.48 & 25.28 & 25.18 \\
\hline \multirow[t]{6}{*}{ Bird } & $10: 1$ & 48.81 & 49.43 & 49.20 & 48.93 & 48.88 \\
\hline & $20: 1$ & 43.63 & 44.31 & 44.11 & 43.89 & 43.70 \\
\hline & $40: 1$ & 39.03 & 39.33 & 39.04 & 38.96 & 38.66 \\
\hline & $60: 1$ & 36.43 & 36.48 & 36.18 & 36.19 & 35.86 \\
\hline & $80: 1$ & 34.62 & 34.58 & 34.23 & 34.34 & 33.95 \\
\hline & 100:1 & 33.19 & 33.19 & 32.79 & 33.00 & 32.56 \\
\hline \multirow[t]{6}{*}{ Bridge } & $10: 1$ & 48.81 & 47.62 & 47.66 & 47.67 & 47.59 \\
\hline & $20: 1$ & 43.63 & 41.96 & 41.88 & 41.92 & 41.81 \\
\hline & $40: 1$ & 39.03 & 36.60 & 36.41 & 36.44 & 36.35 \\
\hline & $60: 1$ & 36.43 & 33.68 & 33.44 & 33.47 & 33.42 \\
\hline & $80: 1$ & 34.62 & 31.78 & 31.53 & 31.52 & 31.44 \\
\hline & 100:1 & 33.19 & 30.43 & 30.19 & 30.11 & 30.00 \\
\hline
\end{tabular}

The visual quality results are shown in figure 2 . The images shown here are compressed at the compression ratio of 100:1 each at decomposition level of 4 , which is optimum level of compression [7]. The results show that wavelet function BIOR2.6, DB2, SYM1 and COIF 1 provides the better compression results in terms of peak signal to noise ratio (PSNR) values in their respective families for the test images.

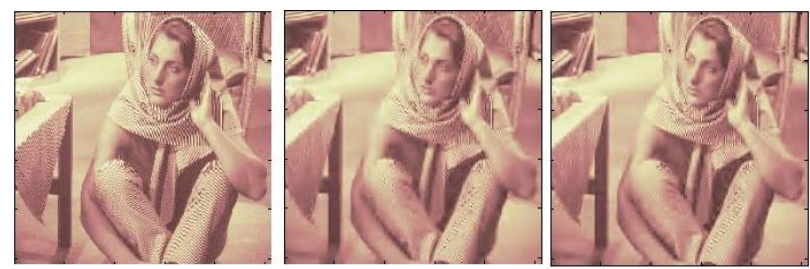

Figure 2: Compression results of image Wbarb (i) original image (ii) reconstructed image using wavelet BIOR 2.2 (iii) reconstructed image using wavelet BIOR 2.6, each at compression ratio 100:1 and decomposition level 5

Also, it is found that the wavelet function BIOR 2.6 gives better compression performance for large size images in terms of PSNR values. While wavelet functions BIOR 2.4 shows the competitive compression performance for the small size images. The analysis and comparison of the results show that the not only in the BIOR family, the wavelet function BIOR 2.6 gives the better compression performance ( in terms of PSNR) in all the wavelet families considered in our experiment. For the compression performance in terms of visual image quality, the wavelet BIOR 2.2 provides the better results for the test image Wbarb and Pepper. While, the wavelet BIOR 2.6 for the images Bird and Bridge gives the better compression performance in terms of visual image quality. This shows that objective as well as subjective quality of the compressed image is better for
Biortogonal wavelet family. Reason behind this performance is that Biorthogonal wavelets can use filters with similar or dissimilar order for decomposition $(\mathrm{Nd})$ and reconstruction $(\mathrm{Nr})$. Therefore Biorthogonal wavelet is parameterized by two numbers and filter length is $\{\max (2 \mathrm{Nd}, 2 \mathrm{Nr})+2\}$ [8]. Also these are Symmetric and Symmetry provides linear phase and minimize border arti-facts.

In study if decomposition level is increased the compression performance improves but the quality of image deteriorates. Further, it is also observed that the BIOR wavelet families take much more computational time in comparison to other wavelet families considered in our experiment. Also it is found that as the filter order increases in a given wavelet family, the compression performance increases, but the visual quality of compressed image becomes poorer. The higher order of filters involves the longer filters, which involves more blurring in the compressed image.

\section{CONCLUSION}

This study presents an analysis and comparison of the wavelet families for image compression considering PSNR and visual quality of image as quality measure. The effects of Biorthogonl, Daubechies, Coiflet and Symlet wavelet families on test images are examined. We analyzed the results for a wide range of wavelet families and found that the wavelet BIOR2.6 provides best compression performance for all variety of images almost at all the compression ratios among all the families considered. The computational time required for the Biorthogonal wavelet family is more in comparison to other wavelet families. As far as the image quality is concerned we got a fair image quality with wavelet BIOR 2.6 at the compression ratio 100:1 and decomposition level 4 for the test images.

\section{REFERENCES}

[1] Talukder, K. H. and Harada, K. 2008. Haar Wavelet Based Approach for Image Compression and Quality Assessment of Compressed Image. IAENG International Journal of Applied Mathematics, 36:1.

[2] Veeraswamy, K. and Srinivas, Kumar S. 2008. An Improved Wavelet Based Image Compression Scheme and Oblivious Watermarking. IJCSNS International Journal of Computer Science and Network Security, 8, 170-177.

[3] Kanvel, T. N. and Monie, E. C. 2009. Performance Measure of Different Wavelets for a Shuffled Image Compression Scheme. IJCSNS International Journal of Computer Science and Network Security, 9, 215-221.

[4] Usevitch, B. E., 2001. A Tutorial on Modern Lossy Wavelet Image Compression: Foundations of JPEG 2000. IEEE Signal Processing Magazine.

[5] Rout, S. 2003. Orthogonal vs Biorthogonal Wavelets for Image Compression. MS Thesis, Virgina Polytechnic Institute and State University, Virgina.

[6] Kharate, G. K., Patil, V. H., Bhale, N. L. 2007. Selection of Mother Wavelet for Image Compression on Basis of Nature of Image. Journal of Multimedia, 12.

[7] Kumari, S. et al. 2010. Performance Analysis of Wavelet Families for Image Compression. Proceedings of National Conference on Advances in Video, Cyber Learning and Electronics, 2010, 24-30.

[8] Kumari, S. et al. 2010. Image Quality Prediction by Minimum Entropy Calculation for Various Filter Banks.', International Journal of Computer Applications, 7(5), 3134. 